\title{
Aprendizagem Social e Comportamentos Agressivo e Lúdico de Meninos Pré-escolares
}

\author{
Social Learning and Aggressive and Playful Behaviors of Pre-school Boys
}

\author{
Timoteo Madaleno Vieira*, ${ }^{*}$, Francisco Dyonisio C. Mendes ${ }^{b}$, \\ \& Leonardo Conceição Guimarães ${ }^{b}$ \\ ${ }^{a}$ Faculdades Alves Faria, Goiânia, Brasil \& ${ }^{b}$ Universidade Católica de Goiás, Goiânia, Brasil
}

\begin{abstract}
Resumo
Investigamos a relação entre fatores de aprendizagem social, acessados via questionários, e os comportamentos agressivos e lúdicos de meninos pré-escolares, através de observação direta durante o recreio. Os participantes foram 15 meninos com idades entre quatro e seis anos, da cidade de Goiânia, GO, Brasil. Uma análise multivariada de variância indicou efeitos significativos de modelos de agressividade em casa nas taxas de agressão durante o brincar. Crianças expostas a punições físicas abusivas, brigas entre adultos e programas violentos de TV apresentaram mais agressões reais. Meninos que relataram brincar com armas de brinquedo em casa não apresentaram mais agressões reais do que os que relataram o contrário, mas apresentaram maior proporção de agressões de faz-de-conta. Os resultados também indicaram que quanto mais modelos agressivos em casa, maior a incidência de comportamentos agressivos.
\end{abstract}

Palavras-chave: Aprendizagem social; Comportamento agressivo; Comportamento lúdico.

\begin{abstract}
We investigated the interaction between social learning factors measured by questionnaires and aggressive and playful behaviors of pre-school children, through direct observation during their playful break time. The subjects were 15 boys between four and six years old who were enrolled in a non-profit child care center in Goiânia-GO, Brazil. A multivariate analysis of variance indicated significant effects of aggressive models at home on aggression levels during playful behavior. Children exposed to abusive physical punishment, adult fighting and violent TV programs engaged in more episodes of aggression during playful breaks. Boys who reported to play with toy guns at home did not engage in aggressive behavior more often than those who did not, but they displayed a higher proportion of pretended aggression. Results also indicated that aggressive behavior becomes more frequent as the number of aggressive models at home increases.
\end{abstract}

Keywords: Social learning; Aggressive behavior; Playful behavior.

Não é por acaso que a agressividade é um tema recorrente nas ciências biológicas e sociais. O comportamento agressivo é uma das conseqüências da inevitável competição por recursos naturais e por parceiros sociais e reprodutivos. Esse tipo de comportamento parece ter sido selecionado por milhões de anos (Lorenz, 1973) e ser comum nas sociedades humanas desde ancestrais muito distantes (Lessa, 2004; Wrangham \& Peterson, 1996).
Apesar de inevitável, a expressão da agressividade depende de uma série de fatores ambientais durante o desenvolvimento do indivíduo. Estudar como comportamentos agressivos são aprendidos e mantidos na infância é fundamental não apenas para a compreensão teórica do fenômeno, como também para sua prevenção. Talvez por isso o volume de pesquisas sobre o comportamento agressivo infantil tem acompanhado o crescimento de problemas comportamentais no contexto social em todo o mundo (e.g. Borge, Rutter, Côté, \& Tremblay, e Weber Martins pela revisão da dissertação que originou o presente trabalho. Ao editor e pareceristas desta revista que prestaram grandes contribuições, com críticas pertinentes e sugestões valiosas. Por fim, nossa gratidão à coordenação do Centro de Educação Infantil Nossa Senhora de Nazaré pelo apoio sem o qual o trabalho não seria possível, e todo nosso carinho às crianças que prestaram a mais importante contribuição de todas ao participarem da pesquisa. 
2004; Maldonado \& Williams, 2005; Strasburger \& Donnerstein, 2005; Vaillancourt, Brendgen, Boivin, \& Tremblay, 2003; Watson, Andreas, Fischer, \& Smith, 2005).

A Teoria da Aprendizagem Social é uma ferramenta teórica importante, já que trata da aprendizagem de novos comportamentos a partir da observação de modelos comportamentais (Bandura, 1979; Bandura, Ross, \& Ross, 1963; Bandura \& Walters, 1963; Barr \& Hayne, 2003; Cloninger, 1999). Tanto características do modelo, tais como o grau de afinidade ou o tipo de papel desempenhado pelo mesmo (figura de um herói, o sexo do modelo, etc.), como as contingências envolvidas na si-tuação, devem ser consideradas. É importante ressaltar que os modelos comportamentais podem se apresentar tanto no comportamento das pessoas do convívio das crianças, como também através de mídias diversas (televisão, videogames, quadrinhos, etc.).

A influência da mídia já vem sendo apontada por diversos autores (e.g. Anderson \& Bushman, 2002; Batista, Fukahori, \& Haydu, 2004; Gomide, 2000, 2002; Macleimont, 2002; Rangel, 2004; Zavaschi, 1998). Contudo, é importante estudar suas influências em conjunto com a influência advinda do ambiente social cotidiano das crianças. Os primeiros modelos com os quais as crianças normalmente interagem são os pais e outros indivíduos significativos do ambiente familiar (irmãos, tios, avós, etc.), e são eles quem normalmente administram o acesso da criança à televisão, cinema, videogames, etc. Além disso, as próprias práticas educativas dos adultos podem ser modelos comportamentais a partir dos quais as crianças aprendem novos comportamentos.

Cecconello, Antoni e Koller (2003) discutem os estilos parentais e as práticas educativas dos mesmos e sua influência sobre o comportamento das crianças. Para esses autores, pais autoritários, que usam a punição física como prática educativa, contribuem para a formação de filhos com problemas comportamentais.

Watson e Peng (1992) observaram crianças pré-escolares norte-americanas em uma situação naturalística, interessados na relação entre o brincar com armas de brinquedo, a punição física e a exposição a modelos de comportamento agressivo da mídia, e cada uma das seguintes categorias: agressividade real (real aggression), agressividade de faz-de-conta (pretend aggression) e brincadeira turbulenta (rough-and-tumble play). Os resultados sugerem que a punição física é o fator mais significativo, dentre os estudados, para a aprendizagem de comportamentos agressivos, bem como sua manifestação disfuncional. A exposição a modelos comportamentais agressivos na mídia apresentou maior relação com agressão de faz-de-conta e brincadeira turbulenta. Já o brincar com armas de brinquedo apresentou relação com as duas categorias de comportamento agressivo, porém, de forma menos significativa em comparação com a punição física.
O estudo simultâneo do comportamento lúdico e o comportamento agressivo, tal qual executado por Watson e Peng (1992), é importante porque nem sempre a separação das duas categorias é clara. Por exemplo, vários estudos nacionais (e.g. Bichara, 2005; Carvalho, Alves, \& Gomes, 2005; Hansen, Macarini, Martins, Wanderlind, \& Vieira, 2007; Rodrigues, 2005) e internacionais (e.g. Jarvis, 2006; Scott \& Panksepp, 2003) sugerem uma relação positiva entre o brincar de forma turbulenta e o desenvolvimento de competências sociais. Desta forma, agredir e brincar vigorosamente (envolvendo interações físicas intensas e que se assemelham a lutas corporais) são categorias comportamentais que parecem representar motivações e funções adaptativas diferenciadas (Lordelo \& Carvalho, 2003). O estudo de Watson e Peng (1992) mostrou que a punição física tende a influenciar o aumento da freqüência de comportamentos agressivos em crianças pré-escolares, ao mesmo tempo em que parece influenciar uma diminuição da ocorrência de episódios de brincadeira turbulenta.

O estudo das duas categorias em conjunto parece, portanto, ser uma boa alternativa para a compreensão tanto do desenvolvimento "normal" como de padrões comportamentais "inadequados" em determinada cultura. Segundo Hansen et al. (2007), parece ser um aspecto universal do comportamento lúdico a representação da forma de interpretar e agir dos adultos da comunidade da qual a criança faz parte. Contudo, Hansen et al. (2007) chamam a atenção para o fato de que as manifestações lúdicas também sofrem influências culturais que não podem ser ignoradas. As representações que aparecem na situação de brincadeira envolvem não apenas a repetição de comportamentos adultos, mas também a construção e reconstrução de significados sociais (Conti \& Sperb, 2001; Santos \& Bichara, 2005).

Chama a atenção o fato de a punição física continuar a ser uma prática educativa bastante utilizada nos dias atuais. Em estudo realizado por Weber, Viezzer e Brandenburg (2004) no Paraná, 88,1\% das 472 crianças participantes relataram já ter sofrido punições corporais. $\mathrm{O}$ que não tem sido comum no Brasil, entretanto, são estudos empíricos, em especial estudos quantitativos com observação direta do comportamento, sobre a relação entre punição física e o comportamento agressivo (bem como estudos sobre a relação entre modelos agressivos na mídia e em casa e o comportamento agressivo). Também não é comum encontrar estudos brasileiros que envolvam a observação do comportamento lúdico e do comportamento agressivo conjuntamente.

O presente estudo teve como principais objetivos investigar a relação entre a punição física, a exposição a modelos comportamentais agressivos (na mídia e no cotidiano familiar) e o brincar com armas de brinquedo, e os comportamentos agressivos e lúdicos das crianças (brincadeira turbulenta e não turbulenta). Desta forma, este trabalho se propõe a verificar se no contexto brasi- 
leiro é possível observar padrões semelhantes aos encontrados por Watson e Peng (1992). A confirmação de tais padrões pode contribuir com a compreensão de fatores universais relacionados ao desenvolvimento infantil, com desdobramentos em fases posteriores do desenvolvimento.

\section{Método}

\section{Participantes}

A amostra inicial incluiu todos os pais ou responsáveis cujos filhos estavam matriculados na instituição $(N=80)$. Todavia, foram selecionados para participar do estudo apenas crianças do sexo masculino, com base na literatura que aponta o repertório comportamental de meninos pré-escolares como tendo maior frequiência de comportamentos agressivos reais e de faz-de-conta, do que o de meninas (Camargo \& Mendes, 2003). Foram utilizados os dados apenas das crianças cujas respostas dadas na entrevista mostraram-se coerentes com as respostas dadas pelos pais ou responsáveis no questionário $(N=$ 20). Destes, apenas 15 tiveram seus dados analisados. Os motivos do descarte dos dados de cinco participantes foram: baixa freqüência às sessões (menos de 10), ou afastamento da instituição, sem possibilidade de localização, antes da entrevista final.

As 15 crianças participantes tinham idade entre quatro e seis anos de idade, e advinham de famílias de classe econômica baixa. Estavam matriculadas em um Centro de Educação Infantil, de natureza filantrópica, da cidade de Goiânia. Também participaram 15 responsáveis (pai, mãe ou responsável legal) por cada uma das crianças.

\section{Procedimento}

Foram administrados questionários aos pais de todas as crianças da creche e verificada a presença de cada um dos fatores aqui estudados. Antes do início da coleta de dados, os pais ou responsáveis foram esclarecidos acerca da natureza do estudo e assinaram o Termo de Livre Consentimento Esclarecido, que autorizava a participação das crianças.

As sessões de observação e registro dos comportamentos ocorreram numa sala de aproximadamente $60 \mathrm{~m}^{2}$, durante períodos de aproximadamente 60 minutos, sempre entre as nove e as dez horas do período matutino, às segundas-feiras e quartas-feiras. Dentro da sala não havia brinquedos ou quaisquer tipos de objetos expostos que pudessem ser manipulados pelas crianças. A ausência de brinquedos se deu como um controle parcial do ambiente de pesquisa, já que a literatura mostra que boa parte dos conflitos entre crianças se dá por disputa por recursos (tais como brinquedos; Camargo \& Mendes, 2003). Participaram das sessões algumas monitoras, instruídas a interferir apenas se a segurança de alguma criança fosse colocada em risco.

A coleta sistemática dos dados se deu ao longo de quatro meses. Durante esta fase, cada pesquisador era res- ponsável pela observação e registro do comportamento de metade das 20 crianças inicialmente selecionadas, sendo 10 para cada observador, observadas simultaneamente. As crianças eram levadas até a sala e evitava-se apresentar qualquer tipo de instrução, visando tornar a situação a mais natural possível. Assim, as interações entre os participantes se iniciavam espontaneamente, como em uma situação de recreio escolar.

\section{Instrumentos}

Questionários. Os questionários ministrados aos pais das crianças continham perguntas fechadas (com cinco alternativas de respostas para cada pergunta) acerca das práticas educativas (se utilizavam ou não punição física; se monitoravam ou não o acesso a programas televisivos; se havia ou não conflitos maritais, etc.). As perguntas foram apresentadas em diferentes versões, com o objetivo de identificar possíveis contradições nas respostas. Para aplicação dos questionários foi convocada uma reunião na instituição onde ocorreu a pesquisa, e pode responder o pai, a mãe, ou algum outro adulto legalmente responsável pela criança.

Observação e Registro. Foram utilizados, em conjunto, os métodos de varredura instantânea (ou scan) e o de todas as ocorrências (Altmann, 1974; Setz, 1991). No scan, a cada três minutos os pesquisadores anotavam em que estado os participantes se encontravam (brincadeira turbulenta, brincadeira não turbulenta, outros). Ao mesmo tempo, durante toda a sessão, pelo método de todas as ocorrências, eram registrados todos os eventos de comportamento agressivo (real ou de faz-de-conta). Para o registro de eventos discriminava-se a categoria comportamental do ator e do receptor, isto é, quem agredia e com qual comportamento agressivo, e quem recebia a agressão e com que comportamento reagia ao ataque.

Um estudo piloto foi realizado para habituar as crianças à presença dos pesquisadores (que não interagiam com as crianças) e para a definição operacional dos comportamentos relevantes (etograma - Lehner, 1979; Martin \& Bateson, 1986). Foi utilizado como modelo base de categorização o estudo de Watson e Peng (1992) sobre comportamentos lúdicos e agressivos infantis. O artigo apresenta três categorias comportamentais distintas: (a) Agressão real (real aggression); (b) Agressão de "faz-de-conta" (pretend aggression); (c) Brincadeira turbulenta (rough-and-tumble play). A categoria agressão real se caracteriza pelo ato de infligir contra um outro indivíduo uma ação que explicitamente possa causar-lhe danos físicos ou psicológicos, independentes da intenção do ator que executa a ação (chutar, puxar cabelo, socar, etc.). Já a categoria agressão de faz-de-conta (pretend aggression) é caracterizada por comportamentos de ameaça e/ou conteúdos simbólicos que simulam um ataque (fingir estar atirando, simulação de artes marciais, desempenho de papel de personagem que agride, etc.). 
A terceira categoria utilizada por Watson e Peng (1992), brincadeira turbulenta (BT), foi definida por Pellegrini (1995) como composta por comportamentos fisicamente vigorosos, agitados e turbulentos (perseguir, empurrar, brincar de luta) acompanhados de afeto positivo das crianças envolvidas na interação. A observação do afeto positivo pode ser realizada pela identificação do riso e outras topografias que ajudam o observador a identificar a aceitação dos envolvidos como sendo aquela uma interação lúdica e não de conflito (Camargo \& Mendes, 2003). A brincadeira turbulenta, de acordo com alguns autores, precisa ser separada das categorias de comportamento agressivo, devido ao fato de ter uma relação com o desenvolvimento de habilidades sociais positivas (Collishaw, Maughan, Goodman, \& Pickles, 2004; Humphreys \& Smith, 1987; Jarvis, 2006; Scott \& Panksepp, 2003; Smith \& Pellegrini, 2004). Finalmente, foi utilizada uma segunda categoria de comportamento lúdico, brincadeira não turbulenta (BNT), caracterizada por brincadeiras que não envolvem categorias de comportamento agressivo ou brincadeira turbulenta (e.g., brincar de cavalinho, dança, trenzinho, etc.). O estudo piloto se encerrou depois de verificada uma coerência de $89 \%$ de acertos entre os registros obtidos, durante uma sessão completa, pelos dois pesquisadores que posteriormente coletariam os dados sistemáticos (TMV e LCG).

Entrevistas com as Crianças Participantes. Com o objetivo de aumentar a fidedignidade, depois de encerrada a fase de observação, cada criança participante foi entrevistada. Foi utilizada uma entrevista semi-estruturada, de aplicação breve (no máximo 10 minutos), com respostas abertas apresentadas pelo experimentadorentrevistador (os entrevistadores foram os mesmos pesquisadores que fizeram a observação direta do comportamento), visando tornar a situação mais adequada à participação das crianças. Foram apresentadas às crianças questões semelhantes às dos questionários administrados aos pais. As respostas das crianças foram comparadas com as respostas dadas pelos pais, para se verificar com maior precisão a presença ou não de cada um dos fatores estudados, no ambiente das crianças.

\section{Resultados}

\section{Análises Preliminares}

Normalidade e Homogeneidade. A análise dos gráficos de distribuição dos valores (histogramas e diagramas de caixa e bigode) revelou uma distribuição ampla e assimétrica das medidas de comportamento lúdico (porcentagem dos registros de brincadeira não turbulenta e de brincadeira turbulenta durante a sessão), e um grande número de valores extremos para vários participantes. As medianas de $B N T$ e $B T$ foram consideradas, portanto, as melhores medidas do comportamento lúdico de cada sujeito ao longo das sessões. Testes estatísticos realizados indicaram que os pressupostos de normalidade e homogeneidade das variâncias foram atendidos para a amostra ( $M$ de Box $=8,72 ; p=0,263$; teste Levene: $F=3,147, \mathrm{gl}=8, p=0,069$ para $B N T ; F=2,598$, $\mathrm{gl}=8, p=0,186$ para $B T$ ), o que permitiu o uso das medianas como variável dependente em uma Análise Multivariada de Variância (MANOVA).

Os pressupostos de homogeneidade e normalidade não foram atendidos no caso das medidas obtidas pelo método de todas as ocorrências (agressões reais e simuladas por hora de observação). Por exemplo, sete participantes não emitiram nenhuma agressão real na maioria das sessões em que estavam presentes, embora alguns deles o tenham feito em taxas relativamente altas em uma ou poucas sessões. Por esses motivos, optou-se por análises não paramétricas para analisar o efeito dos "modelos de agressividade em casa" nestas variáveis (teste $U$ de MannWhitney). Apesar do número alto de testes (quatro fatores por 2 variáveis dependentes), decidiu-se manter o nível de significância como $\alpha=0,05$, já que o número relativamente pequeno de casos (participantes) restringiu o poder do teste.

Grupos das Variáveis. Os comportamentos dos 15 participantes analisados variaram bastante quanto ao número e tipos de modelos de agressividade presentes em suas casas. Uma análise de conglomerados usando o método de agrupamento por média entre grupos, e uma matriz de distâncias binárias (euclidiana ao quadrado), formou inicialmente sete grupos: seis contendo entre dois e três participantes com perfis idênticos quanto aos modelos presentes em suas casas (distâncias entre participantes igual a zero) e um sétimo grupo representado por um único participante (participante B). Os sete agrupamentos iniciais foram posteriormente separados em três conglomerados maiores. O primeiro incluía os dois participantes que não relataram nenhum modelo de agressividade em casa ( $\mathrm{C}$ e $\mathrm{Q})$ e dois participantes que admitiram apenas a presença de armas de brinquedo ( $\mathrm{M} \mathrm{e} \mathrm{T}$ ). O segundo conglomerado contém dois indivíduos expostos a todos os quatro possíveis modelos (E e R), e três expostos a todos exceto armas de brinquedo (N, O e A). Os sete participantes que formam o terceiro grupo relataram a presença de dois ou três modelos em casa: punição e arma (D e L); punição, arma e programas violentos (K, $\mathrm{P}$ e H), ou punição e programas violentos (B).

A variação dos perfis implicou em uma distribuição heterogênea das variáveis dicotômicas do estudo, ou seja, houve bastante variação no número de participantes expostos a cada um dos quatro modelos de agressividade em casa. Cinco participantes relataram brigas em casa. Por outro lado, punição, arma de brinquedo e programas violentos assistidos, quando mensurados de forma dicotômica, foram predominantes na amostra. Apenas quatro crianças indicaram pouca $(n=2)$ ou nenhuma punição em casa (codificados como ausente para a variável dicotômica "puni"). Seis meninos listaram menos do que a mediana de quatro programas violentos (codificados 
como ausente para "tv"); os demais nomearam entre quatro e seis programas violentos. Apenas seis participantes relataram não possuir armas de brinquedo em casa; os demais nove participantes indicaram entre uma e três armas de brinquedo em suas casas (presente para "arma").

\section{Análises Principais}

Influência dos Modelos de Agressividade no Comportamento Lúdico (BT e BNT). Para testar a influência dos modelos de agressividade em casa no comportamento lúdico das crianças, foi realizada uma Análise Multivariada de Variância (MANOVA) através do procedimento GLM do SPSS (v.13.0). Foram utilizadas variáveis dicotômicas que indicavam a presença ou número dos modelos de agressividade em casa. Desta forma, cada fator poderia ter apenas um entre dois valores para cada participante: presença ou ausência de brigas entre adultos, presença ou ausência de armas de brinquedo, muita ou pouca punição, muitos (mais do que três) ou poucos programas violentos de televisão citados. Como variáveis dependentes foram utilizadas as medianas de $B N T$ e $B T$ para cada participante (porcentagem dos registros de brincadeira não turbulenta e de brincadeira turbulenta durante a sessão).
Devido ao tamanho relativamente pequeno da amostra, não foi possível utilizar variáveis discretas ou contínuas como co-variáveis do modelo (e.g. o número exato de programas assistidos, o número total de modelos presentes em casa). Também não foi possível acoplar ao modelo o efeito de interações de todos os pares de fatores. Apesar disto, o procedimento possibilitou analisar os efeitos individuais de cada fator nas variáveis dependentes e o efeito da interação dos dois modelos de agressividade (fatores) cujos efeitos individuais foram significativos.

A MANOVA gerada pelo procedimento GLM indicou um efeito significativo do modelo multivariado para a variável $B T(F(5,9)=5,81 ; p=0,011-$ ver Tabela 1$)$. Juntos, os quatro fatores explicaram $76 \%$ da variância dos valores de brincadeira turbulenta (eta parcial ao quadrado $=0,76)$. A análise dos efeitos univariados entre participantes revelou que esta significância foi devido aos efeitos individuais de dois fatores: presença de armas de brinquedo $(F(1,9)=9,93 ; p=0,012)$ e de brigas entre adultos $(F(1,9)=8,56 ; p=0,017)$. Participantes que relataram estes modelos em casa obtiveram maiores valores de mediana de $B T$ do que participantes que não os relataram (Tabela 2).

Tabela 1

Resultados Univariados da MANOVA Usando Quatro Fatores Dicotômicos

\begin{tabular}{|c|c|c|c|c|c|c|c|c|}
\hline Fonte & $\begin{array}{l}\text { Variável } \\
\text { Dependente } \\
\text { (Mediana) }\end{array}$ & $\begin{array}{l}\text { Soma dos } \\
\text { Quadrados } \\
\text { Tipo III }\end{array}$ & $\mathrm{gl}$ & $\begin{array}{l}\text { Quadrado } \\
\text { Médio }\end{array}$ & $F$ & Sig & $\begin{array}{c}\text { Eta } \\
\text { Parcial ao } \\
\text { Quadrado }\end{array}$ & $\begin{array}{c}\text { Poder } \\
\text { Observado }\end{array}$ \\
\hline \multirow[t]{2}{*}{ Modelo Corrigido } & $B N T$ & 740,96 & 5,00 & 148,19 & 0,49 & 0,78 & 0,21 & 0,12 \\
\hline & $B T$ & 832,49 & 5,00 & 166,50 & 5,82 & 0,01 & 0,76 & 0,89 \\
\hline \multirow[t]{2}{*}{ Intercepto } & $B N T$ & 14609,10 & 1,00 & 14609,10 & 48,04 & 0,00 & 0,84 & 1,00 \\
\hline & $B T$ & 1751,24 & 1,00 & 1751,24 & 61,20 & 0,00 & 0,87 & 1,00 \\
\hline \multirow[t]{2}{*}{ punição } & $B N T$ & 366,22 & 1,00 & 366,22 & 1,20 & 0,30 & 0,12 & 0,17 \\
\hline & $B T$ & 31,32 & 1,00 & 31,32 & 1,09 & 0,32 & 0,11 & 0,16 \\
\hline \multirow[t]{2}{*}{ arma } & $B N T$ & 124,06 & 1,00 & 124,06 & 0,41 & 0,54 & 0,04 & 0,09 \\
\hline & $B T$ & 284,17 & 1,00 & 284,17 & 9,93 & 0,01 & 0,52 & 0,80 \\
\hline \multirow[t]{2}{*}{ briga } & $B N T$ & 101,42 & 1,00 & 101,42 & 0,33 & 0,58 & 0,04 & 0,08 \\
\hline & $B T$ & 244,91 & 1,00 & 244,91 & 8,56 & 0,02 & 0,49 & 0,74 \\
\hline \multirow[t]{2}{*}{ tv } & $B N T$ & 198,81 & 1,00 & 198,81 & 0,65 & 0,44 & 0,07 & 0,11 \\
\hline & $B T$ & 96,60 & 1,00 & 96,60 & 3,38 & 0,10 & 0,27 & 0,38 \\
\hline \multirow{2}{*}{ arma $*$ briga } & $B N T$ & 108,07 & 1,00 & 108,07 & 0,36 & 0,57 & 0,04 & 0,08 \\
\hline & $B T$ & 20,62 & 1,00 & 20,62 & 0,72 & 0,42 & 0,07 & 0,12 \\
\hline \multirow{2}{*}{ Erro } & $B N T$ & 2736,73 & 9,00 & 304,08 & & & & \\
\hline & $B T$ & 257,54 & 9,00 & 28,62 & & & & \\
\hline \multirow[t]{2}{*}{ Total } & $B N T$ & 34435,90 & 15,00 & & & & & \\
\hline & $B T$ & 3790,31 & 15,00 & & & & & \\
\hline \multirow[t]{2}{*}{ Total Corrigido } & $B N T$ & 3477,69 & 14,00 & & & & & \\
\hline & $B T$ & 1090,04 & 14,00 & & & & & \\
\hline
\end{tabular}

Nota. ${ }^{a}$ Calculado para $p<=0,05 ;{ }^{\mathrm{b}} R$ ao quadrado $=0,213(R$ ao quadrado ajustado $=-0,224) ;{ }^{\mathrm{c}} R$ ao quadrado $=0,764(R$ ao quadrado ajustado $=0,632)$. BT $=$ Brincadeira turbulenta; $\mathrm{BNT}=$ Brincadeira não turbulenta. 
Como mostra a Tabela 2, as médias de $B N T$ e de $B T$ foram bastante diferentes para os grupos formados pelos fatores "punição" (muita punição, mais BNT) e "arma" (presença de arma, mais $B T$ ) respectivamente. Por outro lado, o pequeno número de participantes dos grupos formados por estas variáveis representou um cálculo pouco preciso das médias agrupadas, como mostram os valores bastante dispersos dos intervalos de confiança da média.
Todavia, a falta de significância destes testes pode decorrer de erro amostral do tipo I (falha em detectar significância quando ela existe) (Dancey \& Reidy, 2006) e não à falta de efeito dos fatores individuais nas variáveis dependentes. No geral, os testes para detectar significância foram mais poderosos para a variável $B T$ do que para $B N T$ (ver Tabela 1).

Tabela 2

Valores das Brincadeiras Turbulentas e Não Turbulentas, de Acordo com os Valores dos Fatores Dicotômicos

\begin{tabular}{|c|c|c|c|c|c|c|}
\hline \multirow{2}{*}{ Fator } & \multirow{2}{*}{ Valor } & \multirow{2}{*}{$\begin{array}{l}\text { V. Dependente } \\
\text { (Mediana) }\end{array}$} & \multirow{2}{*}{ Média } & \multirow[t]{2}{*}{ E.P. } & \multicolumn{2}{|c|}{ I.C. $95 \%$ da Média } \\
\hline & & & & & Mínimo & Máximo \\
\hline \multirow[t]{4}{*}{ puni } & POUCA & $B N T$ & 31,87 & 11,86 & 5,04 & 58,71 \\
\hline & MUITA & & 49,96 & 8,04 & 31,76 & 68,15 \\
\hline & POUCA & $B T$ & 16,81 & 3,64 & 8,58 & 25,04 \\
\hline & MUITA & & 11,52 & 2,47 & 5,94 & 17,10 \\
\hline \multirow[t]{4}{*}{ arma } & NÃO & $B N T$ & 44,21 & 7,43 & 27,41 & 61,02 \\
\hline & SIM & & 37,61 & 8,24 & 18,98 & 56,25 \\
\hline & NÃO & $B T$ & 9,17 & 2,28 & 4,01 & 14,33 \\
\hline & SIM & & 19,16 & 2,53 & 13,44 & 24,8 \\
\hline \multirow[t]{4}{*}{ briga } & NÃO & $B N T$ & 44,45 & 6,38 & 30,02 & 58,89 \\
\hline & SIM & & 37,37 & 10,20 & 14,30 & 60,45 \\
\hline & NÃO & $B T$ & 8,67 & 1,96 & 4,24 & 13,09 \\
\hline & SIM & & 19,67 & 3,13 & 12,59 & 26,75 \\
\hline \multirow[t]{4}{*}{$t v$} & POUCA & $B N T$ & 50,07 & 8,75 & 30,27 & 69,87 \\
\hline & MUITA & & 44,99 & 5,95 & 31,54 & 58,44 \\
\hline & POUCA & $B T$ & 4,00 & 2,69 & $-2,08$ & 10,09 \\
\hline & MUITA & & 16,44 & 1,83 & 12,31 & 20,58 \\
\hline
\end{tabular}

Nota . Puni $=$ punição física; Arma $=$ Relato de que brinca com arma de brinquedo; Briga $=$ Relato de que é exposto a interações agressivas entre adultos; TV $=$ Relato de que assiste a programas com modelos de comportamento agressivo.

Efeitos dos Modelos no Comportamento Agressivo. A presença de modelos de agressividade em casa apresentou grande influência no comportamento agressivo durante as sessões de observação. As análises univariadas não paramétricas ( $U$ de Mann-Whitney - ver Tabela 3) revelaram efeitos de "punição" e "tv" tanto para a taxa de agressões reais como simuladas e conseqüentemente para a taxa total de agressões por hora. Verificando as saídas de ranks médios, notou-se que a presença de qualquer um dos fatores aumentou os valores das três taxas de agressividade, ou seja, os participantes que relataram "punição" e/ou "tv" em casa agrediram mais freqüentemente seus colegas, tanto de forma "real" como "simulada", do que os que não relataram estes dois fatores.

$O$ teste $U$ de Mann-Whitney também revelou um efeito significativo do fator dicotômico "brigas" (entre adultos) e a taxa de agressões reais, embora este fator tenha tido pouco efeito na taxa de agressões simuladas e nos totais de agressão. A presença de "armas" de brinquedo em casa não influenciou nenhuma das três taxas de agressividade (real, simulada ou total), mas teve efeito significativo na porcentagem de agressões reais do participante (agressão real/total de agressões). Embora os participantes com armas em casa não tenham agredido mais freqüentemente do que os outros participantes, quando agrediam tinham uma tendência maior a fazê-lo de forma simulada, e não real.

A influência acumulativa dos modelos agressivos em casa pode ser vista nos resultados dos testes de correlações não paramétricas da Tabela 4. Os quatro valores de freqüência das punições, o número de programas violentos relatados por cada participante (" $n$ tv viol"), e o número total de modelos aos quais os participantes estavam expostos ("nmodelos") apresentaram correlações positivas moderadas a fortes com as medianas de agressão real, de agressão simulada e conseqüentemente com as medianas do total de agressão. Significância foi alcançada nos índices rho de Spearman para as correlações entre frequiência de punição e agressão real por hora, entre número de programas violentos e agressão simulada por hora e entre as duas variáveis e total de agressão. 
Tabela 3

Resultados das Análises Univariadas Não-Paramétricas

\begin{tabular}{|c|c|c|c|c|c|}
\hline \multirow[t]{2}{*}{ Fator } & \multirow[t]{2}{*}{ Teste Estatístico } & \multicolumn{4}{|c|}{ Variável Dependente } \\
\hline & & $\begin{array}{r}\text { RALHR } \\
(\text { mdn })\end{array}$ & $\begin{array}{r}\text { SIMHR } \\
(\mathrm{mdn})\end{array}$ & $\begin{array}{r}\text { TOTHR } \\
(\mathrm{mdn})\end{array}$ & $\begin{array}{l}\% \text { RAL } \\
\text { (mdn) }\end{array}$ \\
\hline \multirow[t]{3}{*}{ punição } & Mann-Whitney $U$ & 6,0 & 7,0 & 4,0 & 21,5 \\
\hline & $Z$ & $-2,204$ & $-1,978$ & $-2,363$ & $-0,066$ \\
\hline & Significância bicaudal & 0,028 & 0,048 & 0,018 & 0,947 \\
\hline \multirow[t]{3}{*}{ arma } & Mann-Whitney $U$ & 21,5 & 21,5 & 26,5 & 8,0 \\
\hline & $Z$ & $-0,684$ & $-0,655$ & $-0,059$ & $-2,270$ \\
\hline & Significância bicaudal & 0,494 & 0,513 & 0,953 & 0,023 \\
\hline \multirow[t]{3}{*}{ briga } & Mann-Whitney $U$ & 7,5 & 22,5 & 16,0 & 11,5 \\
\hline & $Z$ & $-2,261$ & $-0,309$ & $-1,108$ & $-1,676$ \\
\hline & Significância bicaudal & 0,024 & 0,757 & 0,268 & 0,094 \\
\hline \multirow[t]{3}{*}{$t v$} & Mann-Whitney $U$ & 8,0 & 4,5 & 0,0 & 21,0 \\
\hline & $Z$ & $-2,363$ & $-2,678$ & $-3,199$ & $-0,717$ \\
\hline & Significância bicaudal & 0,018 & 0,007 & 0,001 & 0,474 \\
\hline
\end{tabular}

Nota . Punição = punição física; Arma = Relato de que brinca com arma de brinquedo; Briga = Relato de que é exposto a interações agressivas entre adultos; TV = Relato de que assiste a programas com modelos de comportamento agressivo.

Tabela 4

Correlações Não-Paramétricas Entre os Fatores Medidos de Forma Discreta e as Medidas de Agressividade

\begin{tabular}{|c|c|c|c|c|c|}
\hline & & & $n$ modelos & $n$ tv viol & $\begin{array}{l}\text { frequência } \\
\text { da punição }\end{array}$ \\
\hline Rho de & RALHR (mdn) & Coeficiente & $0,603 *$ & 0,457 & $0,529 *$ \\
\hline \multirow[t]{11}{*}{ Spearman } & & Sig. (bicaudal) & 0,017 & 0,087 & 0,042 \\
\hline & & $N$ & 15 & 15 & 15 \\
\hline & SIMHR (mdn) & Coeficiente & $0,567 *$ & $0,693 * *$ & 0,445 \\
\hline & & Sig. (bicaudal) & 0,027 & 0,004 & 0,096 \\
\hline & & $N$ & 15 & 15 & 15 \\
\hline & TOTHR (mdn) & Coeficiente & $0,676^{*}$ & $0,744 *$ & $0,568 *$ \\
\hline & & Sig. (bicaudal) & 0,006 & 0,001 & 0,027 \\
\hline & & $N$ & 15 & 15 & 15 \\
\hline & $\%$ RAL (mdn) & Coeficiente & 0,034 & $-0,008$ & 0,099 \\
\hline & & Sig. (bicaudal) & 0,905 & 0,976 & 0,725 \\
\hline & & $N$ & 15 & 15 & 15 \\
\hline
\end{tabular}

Nota. $*$ Correlação é significante para $p<=0,05$; ** Correlação é significante para $p<=0,01$. RALHR $=$ Agressões reais por hora; SIMHR = Agressões de faz-de-conta por hora; TOHR = Total de agressões por hora; \%RAL = Percentuais de agressões reais por hora. $N$ modelos $=$ Número de modelos agressivos no ambiente familiar; $N$ TV viol = Número de programas com modelos de comportamento agressivo que a criança relata assistir; Freqüência punição = Freqüência com a qual a criança sofre punição física.

O número total de modelos agressivos presentes em casa (de 0 a 4) foi significativamente correlacionado com estas três medidas de agressividade. Já a porcentagem de agressões reais não mostrou significância em nenhum dos três testes. Em conjunto, a matriz de correlações mostra que tanto um aumento no número de modelos de agressividade aos quais o participante é exposto, quanto um aumento na intensidade de exposição de um único modelo aumenta as taxas de agressão real e simulada, mas não afeta a proporção entre elas. Por outro lado, a freqüência de punição teve um efeito maior sobre a variável agressividade real, enquanto o número de programas violentos citados teve um efeito maior sobre a agressividade simulada. 


\section{Participantes Não Agressivos}

O efeito dos modelos agressivos em casa no comportamento agressivo dos participantes pode também ser ilustrado pela distribuição assimétrica dos modelos de agressividade entre os participantes que geralmente não agrediram durante as sessões (mediana da porcentagem de agressão real igual a zero) e aqueles que exibiram comportamentos agressivos na maioria das sessões. Dos sete participantes que não agrediram ninguém durante a maioria das sessões, três relataram punição e nenhum indicou haver brigas entre adultos em suas casas. Em contraste, todos os oitos participantes que agrediram na maioria das sessões disseram ser punidos freqüentemente em casa e sete admitiram vivenciar brigas entre adultos (teste unicaudal da probabilidade exata de Fisher $=0,026$ e 0,019 respectivamente). Assistir a muitos programas violentos (mais do que três) também mostrou dependência com geralmente agredir ou não durante as sessões (Fisher unicaudal $=0,035$ ). O padrão foi inverso, embora de forma não significativa, para o fator "arma de brinquedo," com mais participantes que geralmente não agrediram durante as sessões indicando a presença deste fator em suas casas (cinco contra quatro; Fisher bicaudal= $0,608)$.

\section{Discussão}

Os resultados apresentaram grande coerência com a Teoria da Aprendizagem Social (Bandura, 1979), bem como com boa parte do trabalho de Watson e Peng (1992). A punição física mostrou uma influência significativa tanto sobre a agressividade real como de faz-de-conta e também apresentou relação com o tipo de brincadeira no qual as crianças se engajaram. As crianças punidas fisicamente se engajaram menos em atividade de brincadeira turbulenta. Partindo das considerações de Jarvis (2006) e Scott e Panksepp (2003) sobre algumas vantagens da brincadeira turbulenta para o desenvolvimento infantil, torna-se relevante apontar que a punição física pode oferecer risco significativo ao desenvolvimento saudável da criança. Essa interpretação dos dados pode levar a pelo menos duas conclusões: (a) A punição física está diretamente relacionada com o aumento da freqüência de comportamentos agressivos, o que significa problemas para o desenvolvimento social da criança no presente e, possivelmente, no futuro, e (b) a punição física está diretamente relacionada com o desinteresse (ou falta de disposição) da criança para engajar-se em um tipo de brincadeira (brincadeira turbulenta) que tem sido apontada como importante para o desenvolvimento de habilidades psicomotoras e sociais. Não se trata de apontar relações de causa e efeito; contudo, os dados mostram uma relação entre punição física e os comportamentos lúdicos e agressivos que sugere que crianças que são punidas fisicamente correm o risco de ter maior dificuldade em desenvolver interações sociais adequadas. Se forem tomadas as considerações de Cecconello et al.
(2003), é possível levantar inclusive a possibilidade de que problemas comportamentais originados na infância continuem na idade adulta.

Os resultados da exposição a modelos comportamentais na TV também foram coerentes com os trabalhos de Batista et al. (2004), Gomide (2000, 2002) e Watson e Peng (1992). Porém, quanto ao trabalho de Watson e Peng (1992), houve uma diferença parcial nos resultados em relação ao presente trabalho, já que naquele os resultados mostraram relação apenas entre TV e agressão de faz-de-conta, sugerindo inclusive "efeito catártico" da exposição a programas com modelos de agressividade na TV. No presente trabalho, os participantes que relataram assistir a um maior número de programas violentos na TV mostraram maior tendência a agredir tanto de forma real como de faz-de-conta, apesar de uma tendência um pouco maior para a agressão de faz-de-conta. Essas diferenças podem ocorrer devido a diferenças culturais que, conforme Morais e Otta (2008) devem ser levadas em consideração no estudo do comportamento infantil. Seria interessante comparar mais dados de estudos especificamente sobre a relação entre TV e comportamento agressivo realizado em diferentes nacionalidades e culturas.

A diferença mais marcante entre os dois trabalhos refere-se ao fator "armas de brinquedos" Em Watson e Peng (1992) o brincar com armas de brinquedo mostrou relação com as duas categorias de comportamento agressivo (somente para crianças do sexo masculino). No presente trabalho o brincar com armas de brinquedo não apresentou relação significativa com nenhuma categoria de comportamento agressivo, tendo sido observada uma relação apenas entre armas de brinquedo e brincadeira turbulenta. Esse resultado levanta a possibilidade da influência de uma possível diferença cultural entre o contexto norte-americano e brasileiro. De acordo com o sociólogo Larkin (2007) existe nos Estados Unidos uma cultura paramilitar que envolve o culto à arma de fogo. O significado da arma para crianças norte-americanas pode ter influenciado os resultados do trabalho realizado naquele contexto cultural.

É interessante apontar que apesar das diferentes interações entre os fatores estudados e as variáveis dependentes, a análise dos dados mostrou que, em conjunto, os fatores aumentaram significativamente o total de agressões. Esse dado também replica resultados do trabalho de Watson e Peng (1992). A arma de brinquedo, sozinha, pode não ser um fator significativo, mas quando combinada a outros fatores, tais como punição física e a exposição a modelos de comportamento agressivo (em especial aqueles que portam armas de fogo), pode se tornar significativo. Outra variável importante a ser considerada é o tipo de intermediação cognitiva presente na apresentação de um modelo agressivo. Segundo Graña et al. (2004), não apenas o modelo em si, mas o modo como se apresenta uma interpretação do mesmo, é fundamental para o tipo de efeito sobre o comportamento das crian- 
ças. Assim, se a arma for apresentada em um contexto lúdico, onde pareça algo inofensivo ou aceitável, e onde as conseqüências negativas do seu uso na cena em questão não estiverem claras (e.g., em desenhos animados ou histórias de super-heróis), o efeito pode ser a aprendizagem do comportamento agressivo e a diminuição da sensibilidade à dor alheia.

Apesar das possíveis variáveis culturais que possam estar influenciando diferenças nos resultados observados em diferentes países, tal como já comentado, chama a atenção o fato de que, por outro lado, alguns resultados muito semelhantes sejam observados em contextos culturais distintos, tomando como referência alguns dos trabalhos que ressaltam a importância de fatores culturais e contextuais para o desenvolvimento humano (e.g., Bichara, 2005; Carvalho et al., 2005; Hansen et al., 2007; Rodrigues, 2005). Tanto no contexto norte-americano como no contexto brasileiro, a punição física aparece como um importante preditor do desenvolvimento de comportamentos agressivos excessivos. Já as diferenças sócio-econômicas pareceram não interferir de forma clara nos resultados, mas isso carece de investigações mais detalhadas. Não se pode afirmar que fatores sócio-econômicos e culturais não sejam relevantes, mas os dados sugerem algumas tendências universais na relação observada especialmente entre a punição física e o comportamento agressivo infantil.

Em ambas as culturas aqui comparadas parece haver uma tendência de que à medida que a frequiência de comportamentos agressivos aumenta, a freqüência de episódios de brincadeira turbulenta diminui (Vieira, 2007; Watson e Peng, 1992). Essa relação pode estar apontando para uma possível incompatibilidade entre as duas categorias comportamentais. Tal como comentado por Rodrigues (2005), no contexto de brincadeira turbulenta não é comum o surgimento de conflitos significativos, sendo mais freqüente o uso de soluções pacíficas. Então, a punição física parece não apenas influenciar o desenvolvimento de padrões comportamentais excessivamente agressivos, como também a diminuição de comportamentos lúdicos como a brincadeira turbulenta, de grande importância para o desenvolvimento da criança (Rodrigues, 2005). Isso chama a atenção para a necessidade de mais divulgação de informações sobre tal prática educativa, já que seu uso parece ser bastante freqüente no Brasil, tal como aponta o trabalho de Weber et al. (2004).

Vale ressaltar que o estudo do comportamento envolve um grande número de variáveis, e fatores culturais podem interferir em níveis diversos (internacionais, regionais, etc.). O trabalho de Morais e Otta (2008) aponta diferenças significativas entre comportamentos de crianças de São Paulo e de uma comunidade praiana (Ubatuba), o que ressalta a complexidade da questão. Portanto, mais dados são necessários para que sejam possíveis conclusões mais precisas.
Vale apontar que há a necessidade de mais investigação e melhoramentos metodológicos. Para futuros estudos, pode-se considerar, por exemplo, o uso de uma amostra significativamente maior e mais representativa. Outrossim, vale explorar a possibilidade de que a segurança das crianças seja garantida sem a presença de adultos (i.e. com o uso de câmeras e monitoramento à distância). Conforme Carvalho et al. (2005), a presença de monitores e professores pode interferir nos padrões de brincadeira. Outra sugestão importante é a de se comparar resultados de estudos realizados em ambientes fechados e ambientes abertos (playgrounds, por exemplo). Por último, é importante alertar que dados empíricos podem prestar mais contribuições se transformados em informações claras e de fácil acesso ao grande público de pais, educadores e possíveis futuros adultos significativos na história de crianças brasileiras e ao redor do mundo.

\section{Referências}

Altmann, J. (1974). Observational study of behavior: Sampling methods. Behaviour, 49, 227-265.

Anderson, C. A., \& Bushman, B. J. (2002). The effects of media violence on society. Science, 295, 2377-2379.

Bandura, A. (1979). Modificação do comportamento. Rio de Janeiro, RJ: Interamericana.

Bandura, A., Ross, D., \& Ross, S. A. (1963). Vicarious reinforcemente and imitation. Journal of Abnormal and Social Psychology, 67, 601-607.

Bandura, A., \& Walters, R. H. (1963). Aprendizaje social y desarrollo de la personalidade. Mexico, DF: Cidade Alianza.

Barr, R., \& Hayne, H. (2003). It's not what you know, it's who you know: Older siblings facilitate imitation during infancy. International Journal of Early Yars Education, 11, 7-21.

Batista, A. P., Fukahori, L., \& Haydu, V. B. (2004). Filme com cenas de violência: Efeito sobre o comportamento agressivo de crianças expresso no enredo de uma redação. Interação em Psicologia, 8, 89-102.

Bichara, I. D. (2005). Apropriação e ressignificação de espaços e equipamentos públicos: Um outro ângulo para o estudo dos contextos de brincadeiras. In L. Souza \& Z. A. Trindade (Eds.), Simpósio Nacional de Psicologia Social e do Desenvolvimento \& X Encontro Nacional do Programa Nacional de Cooperação Acadêmica - Psicologia. Violência e desenvolvimento humano: Textos completos (pp. 28-36). Vitória, ES: Universidade Federal do Espírito Santo.

Borge, A. I. H., Rutter, M., Côté, M., \& Tremblay, R. E. (2004). Early childcare and physical aggression: Differentiating social selection and social causation. Journal of Child Psychology and Psychiatry, 45, 367.

Camargo, J. S., \& Mendes, F. D. C. (2003). Conflitos e sua resolução em crianças de dois a quatro anos de idade. Estudos, Vida e Saúde: Análise do Comportamento e Etologia, 30, 11889-12200.

Carvalho, A. M., Alves, M. M. F., \& Gomes, P. L. D. (2005). Brincar e educação: Concepções e possibilidades. Psicologia em Estudo, 10(2), 217-226.

Cecconello, A. M., Antoni, C., \& Koller, S. H. (2003). Práticas educativas, estilos parentais e abuso físico no contexto familiar. Psicologia em Estudo, 8, 45-54. 
Cloninger, S. C. (1999). Teorias da personalidade. São Paulo, SP: Martins Fontes.

Collishaw, S., Maughan, B., Goodman, R., \& Pickes, A. (2004), Time trends in adolescent mental health. Journal of Child Psychology and Psychiatry, 45, 1350-1362.

Conti, L. D., \& Sperb, T. P. (2001). O brinquedo de pré-escolares: Um espaço de ressignificação cultural. Psicologia: Teoria e Pesquisa, 17(1), 59-67.

Dancey, C. P., \& Reidy, J. (2006). Estatística sem matemática para psicologia. Porto Alegre, RS: Artmed.

Gomide, P. I. C. (2000). A influência de filmes violentos em comportamento agressivo de crianças e adolescentes. Psicologia: Reflexão e Crítica, 13, 1-22.

Gomide, P. I. C. (2002). O efeito de um filme de abuso sexual no comportamento agressivo das adolescentes. Interação em Psicologia, 6, 1-11.

Graña, J. L., Cruzado, J. A., Andreu, J. M., Muñoz-Rivas, M. J., Peña, M. E., \& Brain, P. F. (2004). Effects of viewing videos of bullfights on Spanish children. Aggressive Behavior, 30, 16-28.

Hansen, J., Macarini, S. M., Martins, G. D. F., Wanderlind, F. H., \& Vieira, M. L. (2007). O brincar e suas implicações para o desenvolvimento infantil a partir da Psicologia Evolucionista. Revista Brasileira de Crescimento e Desenvolvimento Humano, 17, 133-143.

Humphreys, A. P., \& Smith, P. K. (1987). Rough and tumble, friendship, and dominance in schoolchildren: Evidence for continuity and change with age. Child Development, 58, 201-212.

Jarvis, P. (2006). "Rough and Tumble" play: Lessons in life. Evolutionary Psychology, 4, 330-346.

Larkin, R. W. (2007). Comprehending Columbine. Philadelphia, PA: Temple University Press.

Lehner, P. N. (1979). Handbook of ethological methods. Cambridge, UK: Cambridge University Press.

Lessa, A. (2004). Arqueologia da agressividade humana: A violência sob uma perspectiva paleoepidemiológica. História, Ciências, Saúde, 11, 279-96.

Lordelo, E. R., \& Carvalho, A. M. A. (2003). Educação infantil e Psicologia: Para que brincar? Psicologia: Ciência e Profissão, 23(2), 14-21.

Lorenz, K. Z. (1973). A agressão: Uma história natural do mal. São Paulo, SP: Martins Fontes.

Macleimont, S. R. Q. (2002). Televisão e crianças: Novas perspectivas de relação. Intercom, 25(1), 11-25.

Maldonado, D. P. A., \& Williams, L. C. A. (2005). O comportamento agressivo de crianças do sexo masculino na escola e sua relação com a violência doméstica. Psicologia em Estudo, 10, 353-362.

Martin, P., \& Bateson, P. (1986). Measuring behaviour. Cambridge, UK: Cambridge University Press.

Morais, M. L. S., \& Otta, E. (2008). Diferenças culturais e de gênero em conflitos pré-escolares. Psicologia: Reflexão $e$ Crítica, 21, 221-232.

Pellegrini, A. D. (1995). School recess and playground behavior. New York: State University of New York.

Rangel, J. G. (2004). Televisão em foco: A violência e suas relações psicossociais no contexto dos telespectadores. $A L$ CEU, 4(8), 51-64.

Rodrigues, M. M. P. (2005). Brincadeira como contexto de desenvolvimento. In L. Souza \& Z. A. Trindade (Eds.), Simpósio Nacional de Psicologia Social e do Desenvolvimento \& X Encontro Nacional do Programa Nacional de Cooperação Acadêmica - Psicologia. Violência e desenvolvimento humano: Textos completos (pp. 43-48). Vitória, ES: Universidade Federal do Espírito Santo.
Santos A. K., \& Bichara, I. D. (2005). Brincadeiras e contextos: Alguns pressupostos para o estudo desta relação. In F. A. R. Pontes, C. M. C. Magalhães, R. C. S. Brito, \& W. L. Martin (Eds.), Temas pertinentes na construção da Psicologia Contemporânea (pp. 277-297). Belém, PA: Editora da Universidade Federal do Pará.

Scott, E., \& Panksepp, J. (2003). Rough-and-tumble play in human children. Aggressive Behavior, 29, 539-551.

Setz, E. Z. F. (1991). Métodos de quantificação de comportamento de primatas em estudos de campo. In A. B. Rylands \& A. T. Bernandes (Eds.), A primatologia no Brasil: Vol. 3 (pp. 411-435). Belo Horizonte, MG: Sociedade Brasileira de Primatologia.

Smith, P. K., \& Pellegrini, A. D. (2004). Play in great apes and humans. In A. Pellegrini \& P. K. Smith (Eds.), The nature of play: Great apes and humans (pp. 285-298). New York: The Guilford Press.

Strasburger, V. C., \& Donnerstein, E. (2005). Children, adolescents, and the media: Issues and solutions. Pediatrics, 103, 129-139.

Vaillancourt, T., Brendgen, M., Boivin, M., \& Tremblay, R. E. (2003). A longitudinal confirmatory factor analysis of indirect and physical aggression: Evidence of two factors over time? Child Development, 74, 1628-1638.

Vieira, T. M. (2007). Fatores de aprendizagem social, comportamento agressivo e comportamento lúdico de meninos pré-escolares. Dissertação de Mestrado não-publicada, Universidade Católica de Goiás, Goiânia.

Watson, M. W., Andreas, J. B., Fischer, K.W., \& Smith, K. (2005). Patterns of risk factors leading to victimization and aggression in children and adolescents. In K. A. KendaallTackett \& S. M. Giacomoni (Eds.), Child victmization: Maltreatment, bullying and dating violenc: Prevention and intervention (pp. 12.1-12.023). Kingston, NJ: Civic Research Institute.

Watson, M.W., \& Peng, Y. (1992). The relation between toy gun play and children's aggressive behavior. Early Education and Development, 3, 370-389.

Wrangham, R., \& Peterson, D. (1996). Demonic males: Apes and the origins of human violence. Boston: Houghton Mifflin.

Weber, L. N. D., Viezzer, A. P., \& Brandenburg, O. J. (2004). O uso de palmadas e surras como prática educativa. Estudos de Psicologia (Natal), 9, 227-237.

Zavaschi, M. L. S. (1998). A televisão e a violência: Impacto sobre a criança e o adolescente. Retrieved November 13, 2006, from http://www.ufrgs.br/psiq/cip.html
Recebido: $25 / 06 / 2008$

$1^{a}$ revisão: $25 / 09 / 2008$

$2^{a}$ revisão: $27 / 07 / 2009$

$3^{a}$ revisão: $21 / 08 / 2009$

$4^{a}$ revisão: $25 / 08 / 2009$

Aceite final: $25 / 08 / 2009$ 